\title{
Surgical results of type I tympanoplasty during the years 2014 to 2019, at the hospital universitario clínica San Rafael in Bogotá
}

\begin{abstract}
Objective: To determine the frequency of postoperative tympanic reperforations at three months and at six months, in patients who underwent type I tympanoplasty in the otorhinolaryngology service of San Rafael University Clinical Hospital in Bogotá, Colombia, during the years 2014 to 2019 .
\end{abstract}

Methodology: Retrospective, descriptive and cross-sectional study.

Study population and methods: The data were collected from the review of otology outpatient medical records, who met the selection criteria and the surgical criteria for institutional type I tympanoplasty.

Results: 47 patients were operated on. $62 \%$ were female and $38 \%$ male. Postoperative reperforation was evidenced in $8.5 \%$ of the total sample. None were before three months, 1 patient between 3 and 6 months postoperative and 3 patients after 6 months postoperative. The most frequent cause for which a patient was led to type I tympanoplasty was infectious, (66\%). The otorhinolaryngological comorbidity most frequently associated with perforations was chronic otitis media (COM), in $51 \%$. The audiometries performed showed an improvement of $17 \%$ and $20 \%$ between the preoperative and postoperative PTA, in the right ear and in the left ear, respectively.

Conclusions: The results show that the type of tympanoplasty used in the institution is effective for the anatomical closure of the tympanic perforations included in our study, despite the associated poor prognostic factors, recommending the use of this surgical technique and this type of cartilaginous graft to achieve this main objective.
Volume 13 Issue 2 - 2021

\author{
Monsalve Murcia DP,' Macias Tolosa $C^{2}$ \\ 'Otorrinolaringóloga Universidad Militar Nueva Granada- \\ Hospital Universitario Clínica San Rafael, Colombia \\ ${ }^{2}$ Especialista en Otorrinolaringología Hospital Universitario \\ Clínica San Rafael, Colombia
}

Correspondence: Daniela Monsalve Murcia, Otorrinolaringóloga Universidad Militar Nueva GranadaHospital Universitario Clínica San Rafael, Colombia, Tel 3045933803,Email danimonsalve9l@gmail.com

Received: March 07, 202I | Published: April 06, 2021

Keywords: tympanoplasty, myringoplasty, tympanic perforation

\section{Introduction}

Tympanic perforations are a frequent otological pathology, with ranges that vary from $0.4 \%$ to $33.2 \%$ in young people and children in developing countries and $1-3 \%$ of the world population. ${ }^{1}$ The damage to the tympanic membrane may be caused by various factors such as explosions, penetrating traumas, barotraumas and infections. The most common symptoms include tinnitus, aural fullness, and hearing loss. ${ }^{2-4}$ In cases where surgical treatment is necessary, tympanoplasties are chosen. The main objective of these procedures is the anatomical closure of the tympanic membrane, reduce recurrent infections and improve hearing thresholds. ${ }^{5}$ The first procedures performed were carried out by Zollner and Wullstein in 1950, which have been modified and adapted to new technologies.

One of the most used techniques is the over under, performed through a microscope, where the graft is placed on the hammer handle and the tympanic remnant below, reported as successful in more than $90 \%$ in various studies. ${ }^{6,7}$ As grafts, different types of tissues are used : fat, perichondrium, cartilage, fascia and dura mater. ${ }^{8}$ Los most commonly used are the fascia of the muscle temporary, the perichondrium tragus and cartilage shell cimba or cavum. Cartilaginous grafts have long been shown to be the most effective for anatomical closure of tympanic perforations independent of underlying anatomical and pathological conditions.

Successful results also depends on other factors as the mechanism of the tympanic perforation, the dysfunction of the Eustachian tube, infections of the upper airway, the age of the patient, the size of the tympanic perforation, comorbidities as otitis media chronic suppurative disease, tympanosclerosis, cholesteatomas, smoking, bilateral perforation and septodeviations. ${ }^{1,2}$ Regarding the audiological results, it is a subject that still generates debate, since its anatomical efficacy has been demonstrated, however, the physiological one is controversial. ${ }^{9}$ Some authors have shown a decrease in hearing gain due to the thickness and rigidity of the cartilage graft. ${ }^{10}$

This type of intervention is also performed in pediatric patients, being an independent group due to anatomical and physiological characteristics. The literature reports higher rates of reperforation, especially in preschool children (under 5 years of age), explained mainly by acute otitis media and recurrent upper respiratory tract infections, immune system disorders and immaturity of the Eustachian tube. ${ }^{11}$ For this reason, some authors propose delaying surgery for at least 8 years, however, there are reports of a greater risk of damage to the inner ear due to prolonged exposure to pro-inflammatory cytokines. ${ }^{12}$ Due to this, the success results are highly variable $(34 \%$ $-94 \%$ ) and there is no consensus on this issue.

What has been established is that the non-repair of tympanic perforations has short and long-term consequences, such as hearing loss, cofosis, chronic otorrhea, external otitis, cholesteatoma, facial paralysis, infections in the nervous system, vertigo, impediments to exposure. getting into the water, impaired quality of life, depression, cognitive decline in adults, and delayed language development in children. 
The objective of this study is to determine the effectiveness of anatomical closure through this technique. Additionally, identify the associated comorbidities, the reasons for which the surgical procedure was decided to be performed, and the sociodemographic characteristics of the intervened population.

\section{Aim}

To determine the frequency of early (three months) and late (six months) postoperative tympanic reperforations, who underwent type I tympanoplasty in the otorhinolaryngology service of the Hospital Universitario Clínica San Rafael de Bogotá, Colombia, during the years 2014 to 2019

\section{Materials and methods}

An observational, retrospective, descriptive, cross-sectional study was conducted by reviewing the medical records of pediatric and adult patients who attended the otorhinolaryngology service of the Hospital Universitario Clínica San Rafael de Bogotá Colombia, during the years 2014 to 2019 and that were operated on by some of the four otologists of the institution. Data were collected from medical records and is evaluaro $n$ postoperative results, as the perforations re post type tympanoplasty I made with the technique over under with cartilage graft and temporal fascia through auricular approach, the timing in which introduced this complication, the size of the perforations, the audiometric results, the most frequently associated comorbidities and causes. For this selection, the inclusion criteria were taken into account: patients of any age with perforation of the tympanic membrane of any etiology, no middle ear or mastoid involvement, patients in whom type I tympanoplasty has been performed using the medial technique over under and those who have achieved postoperative follow-up of at least 6 months, and the exclusion criteria : history of previous otological surgery, concomitant otological surgeries in the same surgical time as type I tympanoplasty or patients in whom no of the medical history to be reviewed.

\section{Statistic analysis}

A multiple correspondence analysis was performed to determine which variables were associated with the presence or absence of postoperative perforations. For the bivariate analysis of the data, the chi-square or Fisher's exact test was used to determine the independence or association between the discrete variables and the presence or absence of postoperative perforations. For the quantitative variables, the means and standard deviations were calculated. Additionally, the statistically significant difference between variables with $p$ values $<0.05$ was established. The selection of the sample was carried out in a non-probabilistic way for convenience. No sample calculation was performed since it was a descriptive study. The collection and database was created in the Microsoft Excel program for subsequent export to the free statistical software R version 5.3 of July 2018 .

\section{Results}

47 patients (47 ears) were operated on, of which 29 were female $(62 \%)$ and 18 male (38\%). These patients had an average age of 36.2 years. Four were under 7 years of age (9\%) See Table 1. Of the total sample of 47 patients, $4(8.5 \%)$ showed postoperative reperforation See Table 2.

Of the patients who underwent reperforations, none were before three months, 1 patient between 3 and 6 months and 3 patients after 6 months postoperatively. See Table 2 . The average time in which the reperforations were documented was 12.8 months. The most frequent cause for which a patient was taken to type I tympanoplasty was infectious, in 31 patients (66\%) and, secondly, traumatic, in 10 patients $(21 \%)$, also being related to a higher probability of reperforation $(p$ $<0.05)$ See Table 2.

The comorbidity most frequently associated with perforations was chronic otitis media (COM), corresponding to 24 patients $(51 \%)$ and non-established in $15(31 \%)$. The OMC, although it was the clinical condition that was most related to postoperative reperforations, showed a $p>0.05$, which was not statistically significant regarding the cause-reperforation relationship.

Regarding the size of the tympanic perforations, there were 26 patients $(55.3 \%$ ) with medium perforations (between $30 \%$ and $60 \%$ ), $15(31.9 \%)$ with large or total perforations (greater than $60 \%$ ) and 6 $(12.8 \%$ ), with small perforations (less than $30 \%$ ). See Table 1. With these results, a correspondence analysis with a Fractionated Chi-sq was performed, finding that large perforations were associated with a higher probability of postoperative reperforation, with a $p<0.05$, being statistically significant in our study. See Graph 1.

Table I Clinical and demographic characteristics of the population with type I tympanoplasty

\begin{tabular}{|c|c|c|c|c|c|}
\hline Variables & & Female & Male & Total & $\%$ \\
\hline \multirow[t]{2}{*}{ Age Group } & $0-7 \mathrm{~A}$ & 2 & 2 & 4 & 9 \\
\hline & >7years & 27 & 16 & 43 & 91 \\
\hline \multirow[t]{8}{*}{ Comorbidity } & OMC & 22 & 2 & 24 & $5 I, I$ \\
\hline & $\begin{array}{l}\text { Not } \\
\text { established }\end{array}$ & 13 & 2 & 15 & 31,9 \\
\hline & Cholesteatoma & 2 & 0 & 2 & 4,3 \\
\hline & OMA & 2 & 0 & 2 & 4,3 \\
\hline & $\begin{array}{l}\text { Sudden hearing } \\
\text { loss }\end{array}$ & I & 0 & I & 2,1 \\
\hline & GOTO & 1 & 0 & 1 & 2,1 \\
\hline & OME & I & 0 & I & 2,1 \\
\hline & Vertigo & I & 0 & I & 2,1 \\
\hline \multirow[t]{3}{*}{ Driiling Size } & Big & 12 & 3 & 15 & 31.9 \\
\hline & Median & 25 & 1 & 26 & 55,3 \\
\hline & Small & 6 & 0 & 6 & 12,8 \\
\hline
\end{tabular}

Table 2 Postoperative results

\begin{tabular}{lllll}
\hline \multirow{2}{*}{ Variables } & \multicolumn{5}{l}{ Reperforación } \\
\cline { 2 - 5 } & No & Si & Total & $\%$ \\
\hline Women & 26 & 3 & 29 & 62 \\
Men & 17 & 1 & 18 & 38 \\
Infectious & 29 & 2 & 31 & 66 \\
Not established & 4 & 2 & 6 & 12.8 \\
Traumatic & 10 & 0 & 10 & 21.3
\end{tabular}

Regarding the audiometric results, these showed an improvement of $17 \%(p=0.20)$ in the preoperative to postoperative PTA, in the right ear and of $20 \%$ in the left ear $(p<0.004)$, the latter being statistically significant in our study. See Table 3 
Table 3 Pre and postoperative auditory tonal averages for Tympanoplasty type I, HUCSR 2014 - 2019

\begin{tabular}{lllll}
\hline \multicolumn{5}{c}{ Auditory Tonal Average dB HL } \\
\hline & Preoperative & Posoperative & Improvement & $\%$ p \\
\hline Right ear & 36,1 & 29,9 & $17 \%$ & 0.2 \\
Left ear & 34,6 & 27,6 & $20 \%$ & $<0.004$ \\
\hline
\end{tabular}

\section{Discussion}

The anatomical closure achieved through the type I over under tympanoplasty technique under a microscope, used at the institution, was $91.5 \%$, which can be considered a good postoperative result according to what has been reported in the national and international literature. In the study carried out by Fernandes et al. In 2018, a closure of tympanic perforations was reported using cartilage as the main graft in $78.3 \%$ to $100 \%$ of patients. ${ }^{11}$ Likewise, Sajid et al. In 2017, demonstrated an anatomical closure success with this technique between approximately $70 \%$ and $80 \% .{ }^{12}$ In the study carried out by Bedoya et al. In 2014, ${ }^{1}$ success rates were even lower than those evidenced in this study, with a success of $53.9 \%$ and failures of $46.1 \%$, results that could be explained by the type of intervened population, very similar to that of our study, with a greater number of comorbidities and difficulties in accessing health services. Likewise, this technique is comparable in terms of effectiveness of anatomical and audiometric closure, with other more innovative ones such as endoscopic tympanoplasty, this one, with effectiveness between $80 \%$ and $100 \% .{ }^{13}$ There was no evidence of a greater risk of perforation in the short term, 3 months, than in the long term, 6 months, in the patients collected.

These data are similar to those reported by Hsern Ern Tan et al. In their meta-analysis, ${ }^{1}$ where no higher incidence of reperforation was found in patients who were followed up at 2 or 12 months, and even suggest that a 6-month postoperative follow-up is sufficient. The use of cartilaginous graft proved to be effective for the closure of perforations in the patients of the institution, even when they had poor prognostic factors (chronic recurrent otitis media, large perforations, bilateral involvement) and even in the absence of another surgical procedure such as mastoidectomy, adenoidectomy, or Tuboplasty. Hsern Ern Tan et al. In 2016, ${ }^{14}$ reported a successful closure in perforations smaller than $50 \%$ when cartilage was used as a graft, with a $2.8 \%$ higher percentage of surgical success, compared with the use of fascia only. Benjamin et al. In $2008,{ }^{15}$ concluded that the performance of mastoidectomy as a concomitant procedure in the same surgical time or later, did not show a lower incidence of postoperative perforations,${ }^{16}$ compared with only tympanoplasty, in patients with pathology uncomplicated (not cholesteatomatous).

A higher prevalence of perforations was evidenced in the female population. This distribution can be related to the greater number of female versus male patients collected in this time cut-off. In the study carried out by Callioglu et al. ${ }^{9},{ }^{1,17}$ no significant difference was found in the prevalence of postoperative reperforations between both sexes. Similarly, Salviz et al. ${ }^{10}$ In $2015,{ }^{18}$ reported a surgical success rate of $72.9 \%$ in men and $79.5 \%$ in women, which may indicate some prevalence by sex of this complication. No higher prevalence of perforations was found in patients under 7 years of age. ${ }^{19}$ Results that agree with those published by Brian et al. In 2010, where they found an efficiency in the closure of perforations between $70 \%$ and $100 \%$, and those of Ryan et al..$^{13}$ In 2016, with a closure percentage between $35 \%$ and $94 \%,{ }^{20}$ which, although lower than in adults, not negligible in terms of effectiveness. They also do not suggest a strict minimum age to perform this type of surgical procedure, however, some authors propose performing this type of surgical procedure, even in patients aged 4 years..$^{21,22}$ In this type of population, the most frequent tympanic perforations are secondary to myringotomy with ventilation tubes, $\mathrm{AOM}$ or trauma. ${ }^{20}$

Regarding comorbidities, chronic otitis media was related to a higher probability of postoperative reperforation, results similar to those reported by Sajid et al. In $2017^{12}$ and by Heo et al. ${ }^{18}$ In $2017,,^{23}$ where it was found that this factor decreases $3.4 \%$ the probability of postoperative closure. Likewise, chronic otitis media was also the main cause for which the tympanic membranes were perforated in our study. The second cause of perforation was traumatic, reported in $21.3 \%$ of patients. This type of mechanism has better prognoses for spontaneous closure and in less time, approximately 4 weeks, ${ }^{24}$ in $80 \%$ to $89 \%$ of cases, and has a lower probability of postoperative reperforations. Some conditions of this are the perforations which engage the hammer or the umbo, are large, compromise periphery (commitment of blood supply), there tympanosclerosis, the edges of the hole are curved or no otorrhea, factors also reduce the surgical success.

In our study, large perforations were associated with a higher probability of postoperative reperforation. These findings are similar to those reported in the 2017 Sajid study, ${ }^{14}$ where it was found that large perforations were re-drilled in $58.3 \%$ of cases, compared to $100 \%$ closure success in small perforations. Likewise, Heo et al. ${ }^{18}$ found ${ }^{14}$ a lower probability, $6.1 \%$, in the postoperative closure of the perforation if it was greater than $50 \%$ and that its location did not have a great influence on the result. Small perforations have a lower incidence of reperforation, since they preserve their anatomical and physiological structure. ${ }^{14}$ Despite the fact that the audiometric results obtained in the study were based solely on the pre and postoperative record of the PTA, not on the closure of the air-bone gap, a statistically significant decrease in the postoperative PTA was evidenced in the ear left most patients, averaging $6.2 \mathrm{~dB}$ in the right ear and $7 \mathrm{~dB}$ in the left ear. Bedoya et al. ${ }^{2}$, obtained an average gain of $14.83 \mathrm{~dB}$ in the evaluated patients. Although the definition of audiometric success is very varied in the literature and there is no consensus, ${ }^{21}$ it is considered a successful result, a postoperative air-bone gap of less than $10 \mathrm{~dB}^{14}$ (26) or a gain of at least $25 \mathrm{~dB}$ postoperatively (27). This shows that an improvement is achieved, not only in the anatomical aspect but also in the auditory one, but that better collection of audiometric data and postoperative follow-up with audiometries of the patients is required to obtain more specific results.

\section{Conclusions}

The results in our study show that the surgical technique used in our institution is effective for the anatomical closure of tympanic perforations in patients without complicated otological pathology, despite the associated poor prognostic factors in the studied population, recommending its use surgical technique and this type of cartilaginous graft with fascia for surgical closure as the main postoperative objective. It was also possible to observe that large 
perforations and having a history of chronic otitis media, which is also the most frequent cause for which this type of surgery is performed, has negative effects on the result of type I tympanoplasty. Age or sex had a negative effect on surgical results in this population group. Regarding the audiometric results, a significant improvement was achieved in the PTA in the left ear, however, it was not possible to follow up the closure of the air-bone gap, the audiometric frequencies involved, or the auditory discrimination due to the lack of data recorded in the medical records, so it is recommended to implement a method of recording these variables in the clinical records used in the otology area of this institution, as well as to carry out clinical and audiometric follow-up of patients in the pre and postoperative period, of at least 6 months

\section{Acknowledgments}

None.

\section{Funding}

None.

\section{Conflicts of interest}

The authors declare that there is no conflict of interest.

\section{References}

1. Tseng CC, Lai MT, Wu CC, et al. Comparison of the efficacy of endoscopic tympanoplasty and microscopic tympanoplasty: A systematic review and meta-analysis. Laryngoscope. 2017;127(8):1890-1896.

2. Bedoya L, Mejía L, Duarte L. Factors related to failed tympanoplasty at La Samaritana University Hospital. Act of Otolaryngology \& Head and Neck Surgery. 2014;42(4):216-221.

3. Elías Ordóñez-Ordóñez L, Vitery Erazo L, Ricardo González Marín N, et al. Tympanoplasty in tympanic perforation secondary to blast wave trauma. Revista Med Universidad Militar Nueva Granda. 2014; 22.

4. Aupy B, Clément P, Crambert A, et al. Shock wave atrial trauma. EMC Otolaryngology. 2013; 42(3):1-9.

5. Lou ZC, Lou ZH, Zhang QP. Traumatic tympanic membrane perforations: A study of etiology and factors affecting outcome. American Journal of Otolaryngology - Head and Neck Medicine and Surgery. 2012;33(5):549555 .

6. Orji FT, Agu CC. Determinants of spontaneous healing in traumatic perforations of the tympanic membrane. Clinical Otolaryngology. 2008;33(5):420-426.

7. Webb BD, Chang; CY Joseph. Efficacy of Tympanoplasty Without Mastoidectomy for Chronic Suppurative Otitis Media. Arch Otolaryngol Head Neck Surg. 2008;134(11):1155-1158.

8. Eliades SJ, Limb CJ. The role of mastoidectomy in outcomes following tympanic membrane repair: A review. Laryngoscope. 2013;123(7):1787802.

9. Callioglu EE, Tijen Ceylan B, Kuran G, et al. Cartilage graft or fascia in tympanoplasty in patients with low middle ear risk index (anatomical and audological results). European Archives of Oto-Rhino-Laryngology. 2013;270(11):2833-2837.
10. Salviz M, Bayram O, Bayram AA, et al. Prognostic factors in type I tympanoplasty. Auris Nasus Larynx. 2015;42(1):20-23.

11. Iacovou E, Vlastarakos PV, Papacharalampous G, et al. Is cartilage better than temporalis muscle fascia in type I tympanoplasty? Implications for current surgical practice. European Archives of Oto-Rhino-Laryngology. 2013;270(11):2803-2813.

12. Yilmaz MS, Guven M, Kayabasoglu G, et al. Comparison of the anatomic and hearing outcomes of cartilage type 1 tympanoplasty in pediatric and adult patients. European Archives of Oto-Rhino-Laryngology. 2015;272(3):557-562

13. Ryan MA, Kaylie DM. What is the optimal age to repair tympanic membrane perforations in pediatric patients? Laryngoscope. 2016;126(10):2201-2202.

14. Lagos A, Villarroel P, García-Huidobro F, et al. Tympanoplasty: factors associated with anatomical and audiometric results. Acta Otorrinolaringologica Espanola. 2020;71(4):219-224.

15. Darouassi Y, Aljalil A, Ennouali A, et al. Prognostic factors of myringoplasty: Study of a 140 cases series and review of the literature. Pan African Medical Journal. 2019;33:323.

16. Iacovou E, Vlastarakos P v., Papacharalampous G, Kyrodimos E, Nikolopoulos TP. Is cartilage better than temporalis muscle fascia in type I tympanoplasty? Implications for current surgical practice. European Archives of Oto-Rhino-Laryngology. 2013;270(11):2803-2813.

17. Lin YC, Wang WH, Weng HH, et al. Predictors of Surgical and Hearing Long-term Results for Inlay Cartilage Tympanoplast. Arch Otolaryngol Head Neck Surg. 2011;137(3):215-219.

18. Heo KW. Outcomes of type I tympanoplasty using a cartilage shield graft in patients with poor prognostic factors. Auris Nasus Larynx. 2017;44(5):517-521.

19. Fernandes VLG, Goel HC, de Sousa E, et al. A Comparative Study of Type-I Underlay Tympanoplasty with Temporalis Fascia Graft Alone and with Conchal Cartilage. Indian Journal of Otolaryngology and Head and Neck Surgery. 2019;71(Suppl 1):1320-1326

20. Gao T, Li X, Hu J, et al. Management of traumatic tympanic membrane perforation: A comparative study. Therapeutics and Clinical Risk Management. 2017;13:927-931.

21. Shah MI, Ghani R, Asif M. Type-I Tympanoplasty By Underlay Technique - Factors Affecting Outcome. Journal of Ayub Medical College, Abbottabad: JAMC. 2017;29(2):258-261.

22. Tan HE, Santa Maria PL, Eikelboom RH, et al. Type I Tympanoplasty Meta-Analysis: A Single Variable Analysis. Otology and Neurotology. 2016;37(7):838-846.

23. Hardman J, Muzaffar J, Nankivell P, et al. Tympanoplasty for Chronic Tympanic Membrane Perforation in Children: Systematic Review and Meta-analysis. Otology \& Neurotology. 2015;36(5):796-804.

24. Babu S, Luryi AL, Schutt CA. Over - under versus medial tympanoplasty: Comparison of benefit, success, and hearing results. Laryngoscope. 2019;129(5):1206-1210. 